\title{
The long reach of liver transplantation
}

\author{
Thomas E Starzl
}

\begin{abstract}
After graduating from high school in my hometown of Le Mars, Iowa, I had a stint in the US Navy (1944-1945), earned a BA degree from Westminster College in Missouri (1947) and completed two years of medical school at Northwestern University (1947-1949). I then dropped out to do research in neurophysiology under the inspiring tutelage of Horace W. Magoun, first at Northwestern and subsequently at the then-new medical school of the University of California, Los Angeles. With MD and PhD degrees from Northwestern, I served a one-year internship at Johns Hopkins Hospital before doing a full-time, 18-month study of complete heart block, which was a frequent complication with the first human open-heart operations. The experiments involved creation of a canine model of heart block and treatment of its adverse consequences with repetitive low-voltage ventricular stimulation-the first epicardial pacemaking.
\end{abstract}

\section{The experimental transplant models}

After I returned to my duties as a surgical resident (1954-1959), discussions with surgical colleagues about the liver's double-blood supply prompted my spare-time development of canine models of abdominal organ transplant. The question was whether hormone- and nutrientrich portal blood was important for optimal total-body metabolism and, specifically, for liver health. This subject had been unresolved for nearly 80 years because of the confusing literature on portacaval shunt (Eck's fistula). The canine model of auxiliary liver transplantation described in 1955 by C. Stuart Welch at Albany Medical College epitomized the controversy. While leaving the blood supply of the native liver intact, Welch arterialized the extra liver (an allograft) but provided its portal inflow with systemic venous return from the lower part of

Thomas E. Starzl is Director Emeritus, Thomas E. Starzl Transplantation Institute, Professor of Surgery, Distinguished Service Professor, University of Pittsburgh, Pittsburgh, Pennsylvania, USA. e-mail: mangantl@upmc.edu the body (Fig. 1a). The dramatic shrinkage of the transplanted liver was attributed to rejection. My alternative explanation was that the allograft had been deprived of liver-supporting (hepatotrophic) factors in the portal blood.

In 1958-1959, I developed two procedures to test this hypothesis. One was liver replacement ${ }^{1}$ (Fig. 1b). The other was removal of the liver and all the other intra-abdominal organs and replacement of them with a multivisceral allograft $^{2}$ (Fig. 1c). The three models in combination generated parallel avenues of research. The first concerned the metabolic crossregulation of the different abdominal organs and, in particular, the importance of their relative anatomic positions. By showing that endogenous insulin and other molecules in portal blood play a crucial part in the control of the liver's size, ultrastructure, function and capacity for regeneration ${ }^{3}$, the hepatotrophic research contributed importantly to the scientific basis of liver transplantation and filled in present and future gaps throughout hepatology and regenerative medicine.

\section{Human organ transplantation}

Despite the intrinsic scientific interest of the transplant operations, their use as a treatment for human liver diseases was the dominant theme in my laboratory and in clinical research from 1958 onward. Unbeknownst to me, 1958 also marked the start of similar efforts by Francis D. Moore at the Peter Bent Brigham Hospital (now Brigham and Women's Hospital) in Boston. At the time, the only evidence that rejection might be avoidable came from epochal experiments done by Rupert Billingham, Leslie Brent and Peter Medawar in 1953, in which allogeneic spleen cells were transplanted into immunologically immature mice. This model presaged bone marrow transplantation in immunodeficient humans. In a second model, which heralded clinical bone marrow transplantation for a wide range of other indications, Joan M. Main and Richmond T. Prehn reduced the immune responsiveness of adult mice with irradiation before transplanting donor lymphoid cells. In both mouse models, animals permanently bearing donor cells (donor leukocyte chimerism) could accept tissues from the original donor but not from others (donor-specific tolerance).

The feasibility of the mouse tolerance models required a close match between donor and recipient tissue (histocompatibility) antigens. Otherwise, the donor cells would be rejected or cause graft-versus-host disease. Because it was another 15 years before enough human leukocyte antigens had been discovered to permit donor-recipient matching, clinical bone marrow transplantation was not accomplished until 1968. In contrast, during the 1959-1962 time frame of my liver model development, kidney allografts were successfully transplanted in seven sublethally irradiated human recipients, without tissue matching and in the ostensible absence of donor leukocytes (Table 1). These patients were rare exceptions to the usual outcome of transplant rejection and patient death, but they made the concept of liver replacement in humans seem less remote.

In the next step, Joseph Murray at Peter Bent Brigham transplanted a kidney into a patient who had not received irradiation and achieved graft function for 17 months under daily treatment with azathioprine, a drug that Roy Calne (with Murray) and Charles Zukoski (with David Hume in Richmond, Virginia) had preclinically tested. Enthusiasm for such pharmacological immunosuppression waned when the case was an isolated success. By then (early 1962), I had obtained a supply of azathioprine for evaluation alone or with other agents in canine transplant models. When we stopped treatment at 100 days in the dogs that had survived that long, many of the liver recipients and an occasional kidney recipient did not reject their grafts. Although rare, these precious dogs contributed to the confidence with which we launched human kidney and liver programs (in that order) at the University of Colorado, Denver. However, more substantive reassurance 


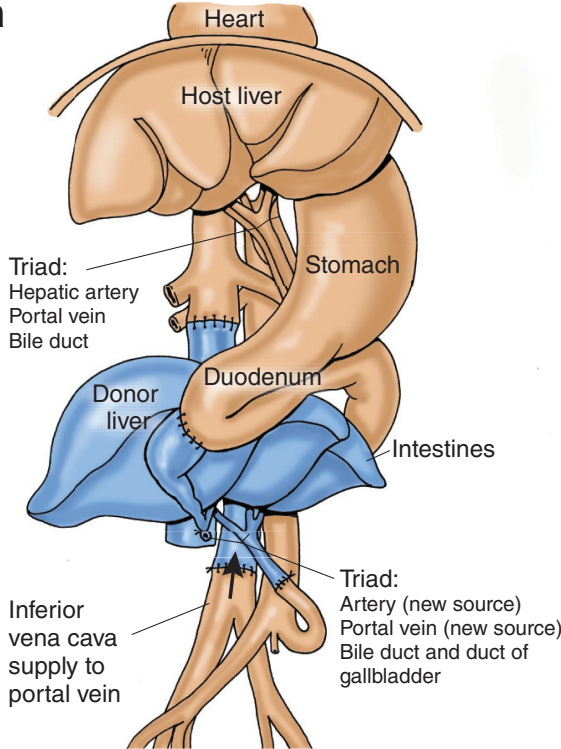

b

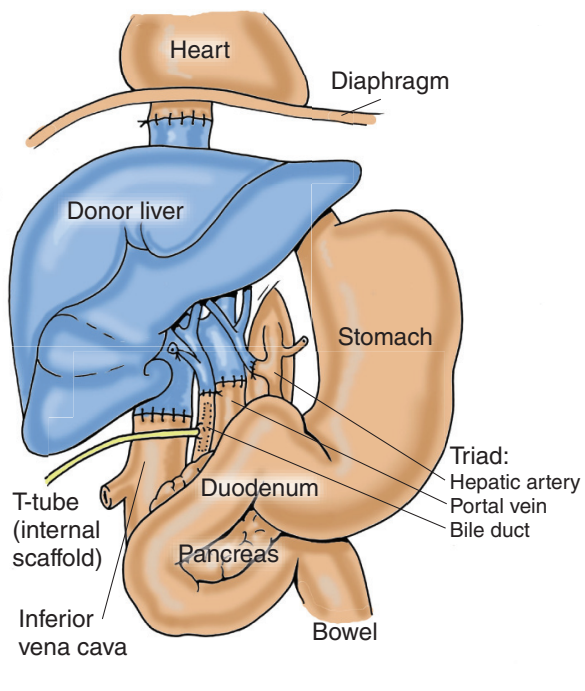

C

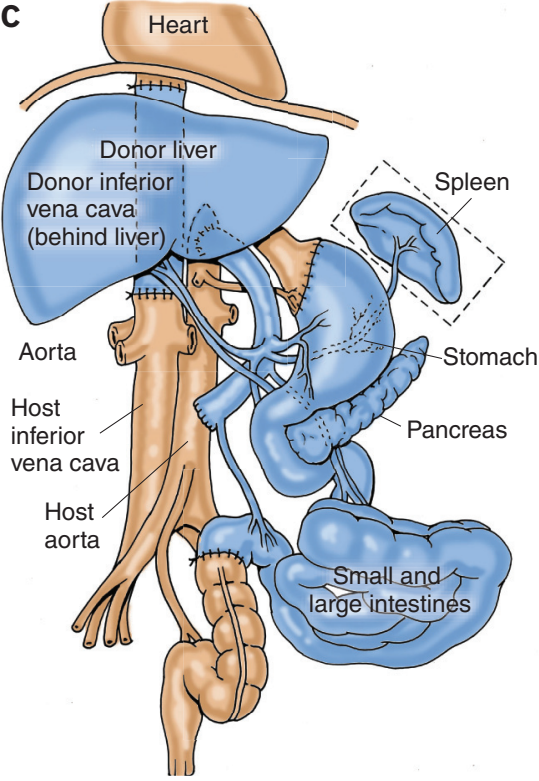

Figure 1 Three early approaches to liver transplantation. (a) Welch's auxiliary liver transplantation in dogs. (b) Complete liver replacement in dog. The fact that the recipient was a dog rather than a human is identifiable only by the multi-lobar appearance of the liver. (c) Organs (blue) of a multivisceral graft in dogs or humans. Illustration by Jon Coulter, M.A., C.M.I.

came from our reproducible observations in dogs, which had not been apparent under the testing conditions in other laboratories.

We used the Denver dog findings to design clinical protocols. We started human recipients of kidneys from living donors on a daily azathioprine regimen several weeks before transplantation, adding prednisone afterward to treat the rejections that almost invariably occurred. This incremental use of drugs exposed two features of the immune system that were generalizable to all kinds of transplanted organs. These features made up the title of a report, published in the October 1963 issue of Surgery, Gynecology and Obstetrics (now the Journal of the American College of Surgeons), of the world's first series of repetitively successful kidney transplantations: "The reversal of rejection in human renal homografts with subsequent development of homograft tolerance." Tolerance was inferred from a declining need for treatment after rejection reversal. For example, the third patient (Fig. 2a) is now the world's longestsurviving kidney allograft recipient, at 49.5 years after transplantation, and has been immunosuppression free for the past quarter-century.

When that series was inaugurated in 1962, Murray's long-standing kidney-transplant program at the Brigham was the only clinically active one in North America. Guided by freely shared data from the University of Colorado, David Hume established a third clinical program, at the Medical College of Virginia (now Virginia Commonwealth University), in which azathioprine and prednisone were also used. During the next two years, nearly 50 renal centers were founded or were gearing up in the United States, and similar events were occurring in Europe. The development of new centers was facilitated by my 1964 textbook Experience in Renal Transplantation, which was based on our lab experiments and research in humans.
Swept up by the momentum of our successes with kidneys, we attempted five human liver replacements between March and October of 1963, using the same immunosuppression but foreshortening pretreatment ${ }^{4}$. Maximum survival was 23 days. All but one of the hepatic grafts functioned throughout, and we found little evidence at autopsy of rejection or preservation injury. Instead, death was caused by infections at multiple sites. In the lung, these were associated with pulmonary emboli, which formed in and migrated from the venovenous bypasses that had been an essential component of the canine liver operation. After two more failed attempts, one in Boston (by Moore) and the other in Paris, all human liver-transplant activity ceased worldwide until the summer of 1967. The procedure had come to be perceived as too difficult to ever be tried again.

During the moratorium, we addressed problems that contributed to the 1963 failures,

Table 1 Characteristics of the first successful transplantations of kidney allografts with $\geq 6$ months survival as of March 1963

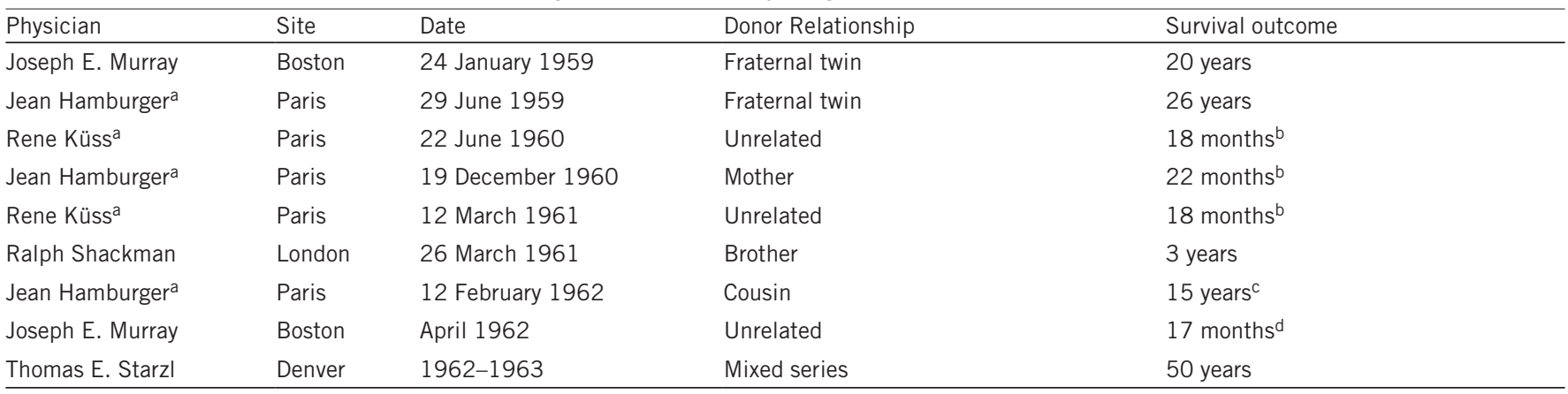

${ }^{a}$ Küss and Hamburger described periodic administration of adrenal cortical steroids with these patients. ${ }^{b}$ Patient death occurred at listed time. ${ }^{\mathrm{C} P a t i e n t}$ underwent successful retransplantation in the 1970's. 'First success with drugs-only immunosuppression (no radiation). 

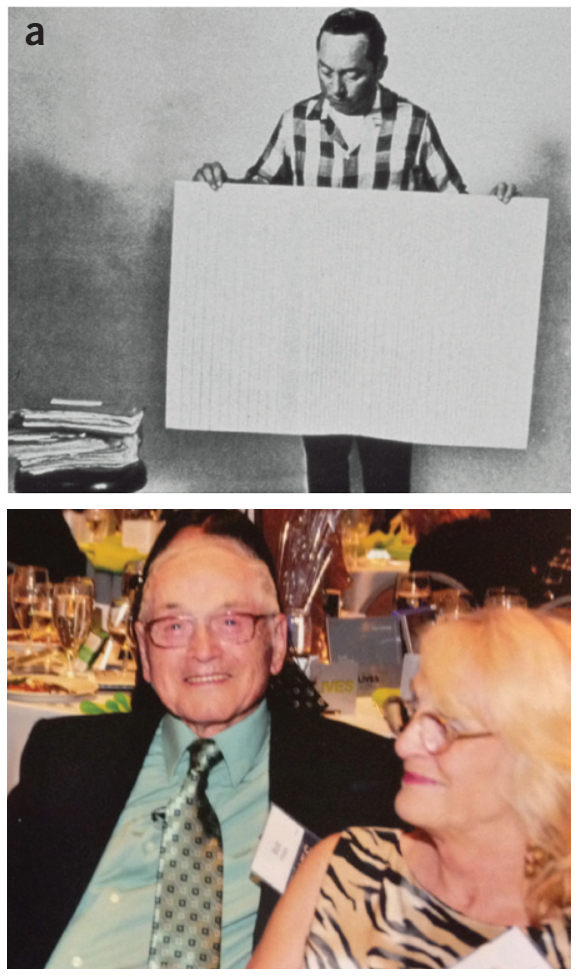

Figure 2 Early successes of liver transplantation. (a) The world's longest-surviving renal-allograft recipient at age 37 (top) and 86 (bottom) years. (b) The Swedish surgeon Carl Groth with three long-surviving liver recipients in Denver, 1967. (c) The world's longest-surviving liver recipient, before (top left), shortly after (top right) and 42.7 years after (bottom) transplantation.

achieving control of blood coagulation, improved organ preservation, infection containment and avoidance when possible of venovenous bypasses. To improve immunosuppression, we refined antilymphocyte globulin from the serum of horses immunized against human lymphoid cells for use as an adjunct to azathioprine and prednisone. Equally important, livergraft availability was enhanced by the evolving general acceptance of brain death. Beginning in July 1967, multiple liver recipients survived for longer than one year (Fig. 2b) with the triple immunosuppression regimen of azathioprine, prednisone and antilymphocyte globulin ${ }^{5}$.

Over the next two years, my colleagues and I achieved enough successes to publish the 1969 Experience in Hepatic Transplantation ${ }^{6}$, a companion textbook to my 1964 kidney book. By then, the first successful human heart transplants (in Cape Town, South Africa, and Palo Alto, California) and pancreas transplants (in Minneapolis) had been recorded, all using the three-drug immunosuppression regimen. However, a dozen more years passed before the promise of liver and other kinds of non-renal transplantation was fulfilled. Even so, four European liver centers were founded during this interval: the first by Roy Calne (Cambridge, UK) in 1968, followed by Henri Bismuth (Paris), Rudolf Pichlmayr (Hannover, Germany) and Rudi Krom (Gronigen, The Netherlands) during the 1970s. Much of the framework of liver
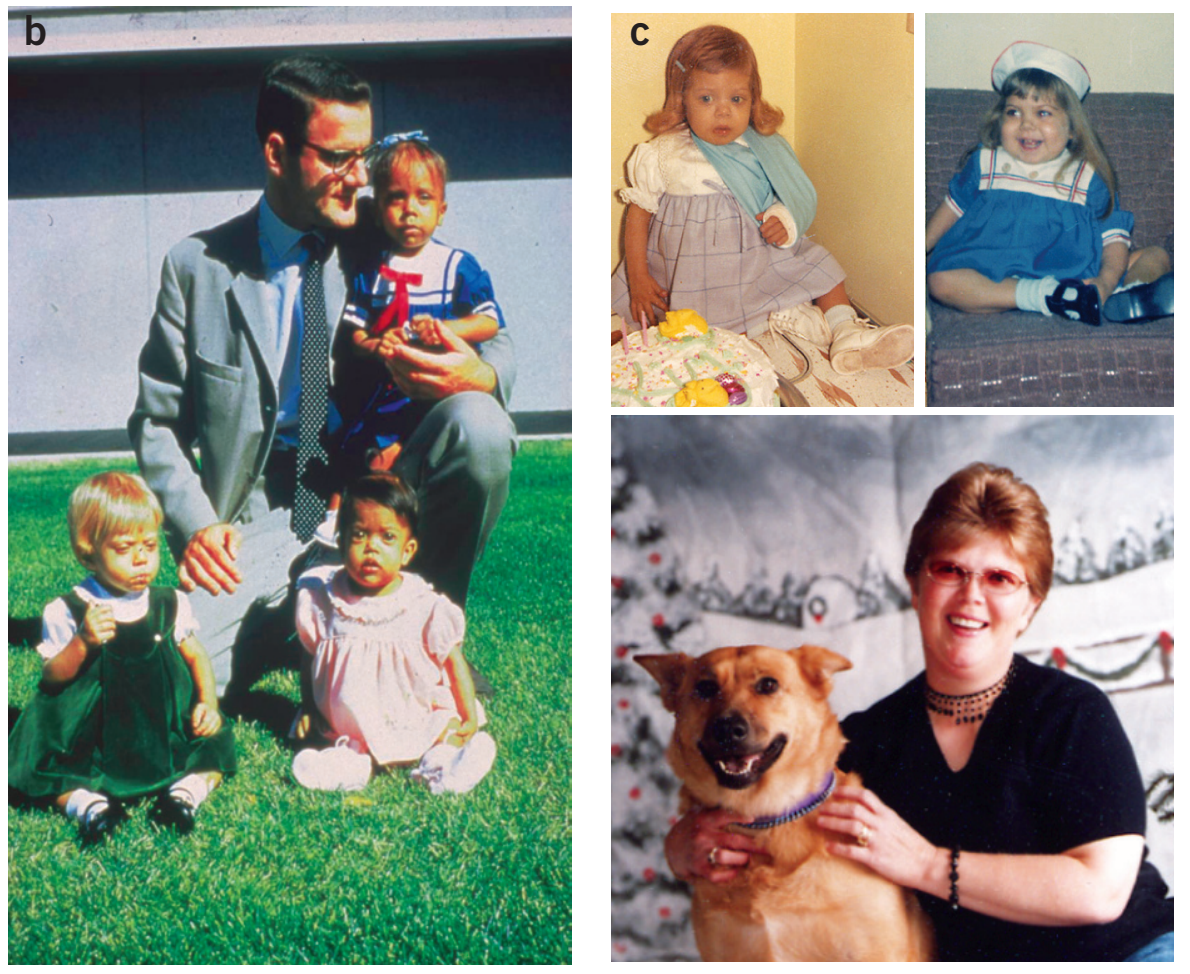

transplantation in place today was developed through the transatlantic alliance of these five centers. All the while, the continued survival of brave forerunner liver recipients kept hope alive. The world's longest-surviving recipient, who was treated for biliary atresia as a child, has now borne her transplanted liver for 42.7 years (Fig. 2c).

Most of the indicators of transplant candidacy were obvious, including inheritable disorders with known liver-based biochemical explanations (for example, Wilson's disease). The litmus test of liver transplantation itself ultimately helped to elucidate the mechanisms and pathophysiology of some inborn errors of metabolism that were not as well understood: $\alpha$-1-antitrypsin deficiency, glycogen-storage disorders and hyperlipoproteinemia. However, liver transplantation remained 'feasible but impractical' until the advent of cyclosporine. In 1979, Roy Calne reported its use in 34 patients who had received transplants, two of whom were liver recipients. Cyclosporine was far more potent than azathioprine. However, its side effects caused its near abandonment until we combined it with prednisone in our original two-drug protocol.

Kidney recipients were the first to benefit from our cyclosporine-prednisone regimen. Close behind, 11 of our first 12 liver recipients, treated during 1979-1980, survived for longer than one year ${ }^{7}$. In December of 1980, I moved from Colorado to Pittsburgh, where my colleagues and I established efficacy of the treatment for other transplanted vital organs. In December of 1981, the promising developments were reported to C. Everett Koop, who took office as US Surgeon General the following January. With personal and sustained encouragement from then-president Ronald Reagan, Koop initiated steps leading to a Consensus Development Conference for liver transplantation that would be held by the US National Institutes of Health and include input from the four European centers.

On 23 June 1983, the consensus committee concluded that liver transplantation had become a "clinical service" rather than an

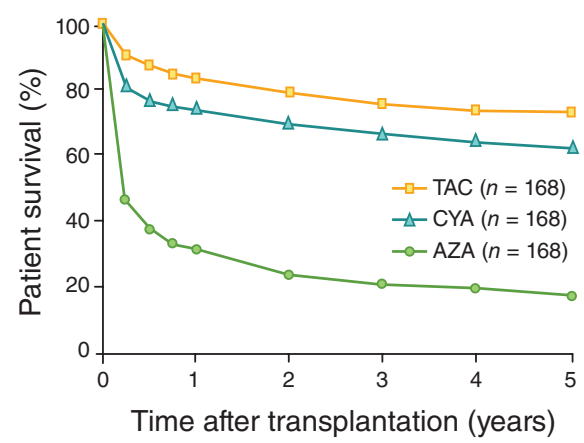

Figure 3 Stepwise immunosuppressionassociated improvements in liver-transplant survival. Similar improvements subsequently occurred with other organs. AZA, azathioprine; CYA, cyclosporine; TAC, tacrolimus. 

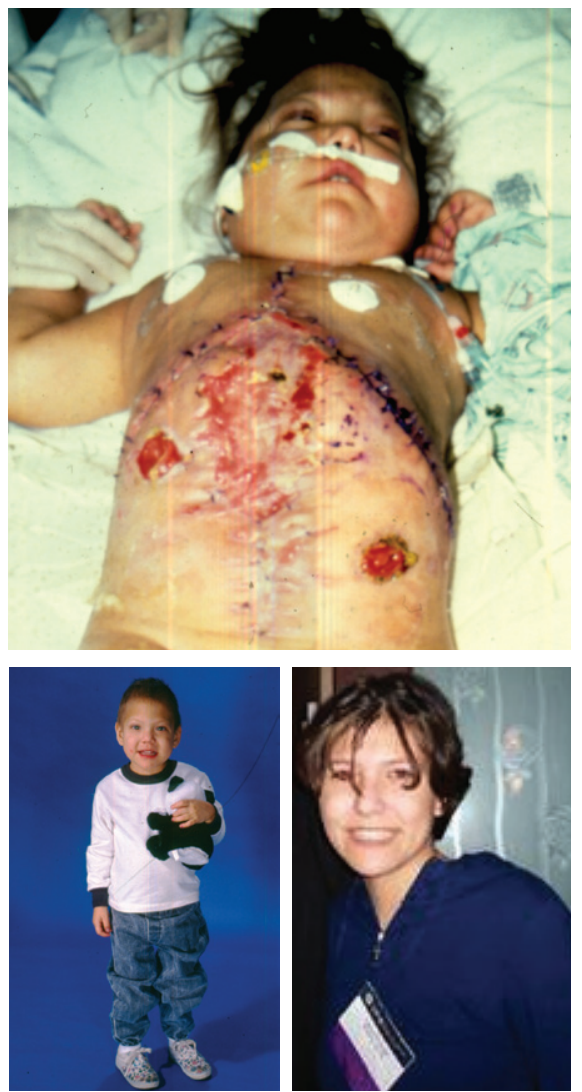

Figure 4 A multivisceral transplant recipient at three stages of her life.

experimental procedure. The resulting worldwide stampede to develop liver-transplant centers was even more dramatic than that of kidney transplantation two decades earlier. In 1989, only six years later, a 17-page article divided between the 12 and 19 October issues of the New England Journal of Medicine began with the following statement: "The conceptual appeal of liver transplantation is so great that the procedure may come to mind as a last resort for virtually every patient with lethal hepatic disease."

Meanwhile, at our lab in Pittsburgh, we had set in motion preclinical studies of tacrolimus ${ }^{8}$ that eventually led to its substitution for cyclosporine and fast-track approval by the US Food and Drug Administration. With tacrolimus, there were further improvements in survival with liver (Fig. 3) and, ultimately, all kinds of organ transplantation. In addition, tacrolimus elevated the liver-inclusive multivisceral transplant procedures developed more than three decades earlier in dogs to the status of "clinical service." The world's longest-surviving multivisceral recipient, now a school teacher, received her transplant 22 years ago (Fig. 4).

\section{The exegesis of allo-engraftment}

My colleagues and I made a connection between organ allo-engraftment and the mouse models of acquired tolerance in 1992, when we discovered a small multilineage population of donor leukocytes (microchimerism) in our long-surviving recipients of liver, kidney and other organs. We found that organ engraftment could be explained by the "...spread of the transplanted organ's lymphoid and dendritic cells through vascular routes to host lymphoid tissues," with induction of "... responses of coexisting donor and recipient immune cells, each to the other, causing reciprocal clonal expansion, followed by peripheral clonal deletion"9 (Fig. 5). In this view, organ transplantation was a form of donor leukocyte chimerism-dependent tolerance, the completeness of which could be inferred from the amount of maintenance immunosuppression required.

Contemporaneously, Rolf Zinkernagel (Zurich) formally proved that the specific $\mathrm{T}$ cell response against noncytopathic microparasites could be exhausted and deleted, and he invoked clonal deletion and 'immune ignorance' as the mechanisms of tolerance to intracellular pathogens, which manifests clinically as a diseasecarrier state. Immune ignorance refers to the failure to recognize the presence of an antigen that does not reach host lymphoid organs; it was first described in the context of transplantation in 1967 by Clyde Barker and Rupert Billingham. On the premise that donor leukocytes and intracellular pathogens were mobile antigen equivalents that could induce clonal activation, exhaustion and deletion, Zinkernagel and I described a spectrum of transplantation scenarios, from outright rejection to durable tolerance, and their infection analogs ${ }^{10}$.

Our generalizable conclusion was that "...migration and localization of antigen are the governing factors in the immunologic respon- siveness or unresponsiveness against infections, tumors, and self and against xenografts and allografts." In this view, all immunological outcomes are determined by the balance between the quantity of antigen with access to host lymphoid organs and the number of antigenspecific $T$ cells induced at these lymphoid sites. Moreover, the fundamental role of transplant immunosuppression (irradiation, drugs and antilymphoid antibodies) is to tilt and maintain a balance favoring donor leukocyte supremacy. With this insight, it has been possible to analyze what has been and what might be accomplished to reduce or eliminate the need for lifetime immunosuppression in transplant recipients.

\section{COMPETING FINANCIAL INTERESTS}

The author declares no competing financial interests.

1. Starzl, T.E., Kaupp, H.A. Jr., Brock, D.R., Lazarus, R.E. \& Johnson, R.V. Reconstructive problems in canine liver homotransplantation with special reference to the postoperative role of hepatic venous flow. Surg. Gynecol. Obstet. 111, 733-743 (1960).

2. Starzl, T.E., Kaupp, H.A. Jr., Brock, D.R., Butz, G.W. Jr. \& Linman, J.W. Homotransplantation of multiple visceral organs. Am. J. Surg. 103, 219-229 (1962).

3. Starzl, T.E. et al. The origin, hormonal nature, and action of hepatotrophic substances in portal venous blood. Surg. Gynecol. Obstet. 137, 179-199 (1973).

4. Starzl, T.E. et al. Homotransplantation of the liver in humans. Surg. Gynecol. Obstet. 117, 659-676 (1963).

5. Starzl, T.E. et al. Orthotopic homotransplantation of the human liver. Ann. Surg. 168, 392-415 (1968).

6. Starzl, T.E. Experience in Hepatic Transplantation (WB Saunders Company, Philadelphia, PA, 1969).

7. Starzl, T.E., Klintmalm, G.B.G., Porter, K.A., Iwatsuki, S. \& Schroter, G.P.J. Liver transplantation with use of cyclosporin A and prednisone. N. Engl. J. Med. $\mathbf{3 0 5}$ 266-269 (1981).

8. Starzl, T.E. et al. FK 506 for human liver, kidney and pancreas transplantation. Lancet 334, 1000-1004 (1989).

9. Starzl, T.E. et al. Cell migration, chimerism, and graft acceptance. Lancet 339, 1579-1582 (1992).

10. Starzl, T.E. \& Zinkernagel, R. Antigen localization and migration in immunity and tolerance. N. Engl. J. Med. 339, 1905-1913 (1998).

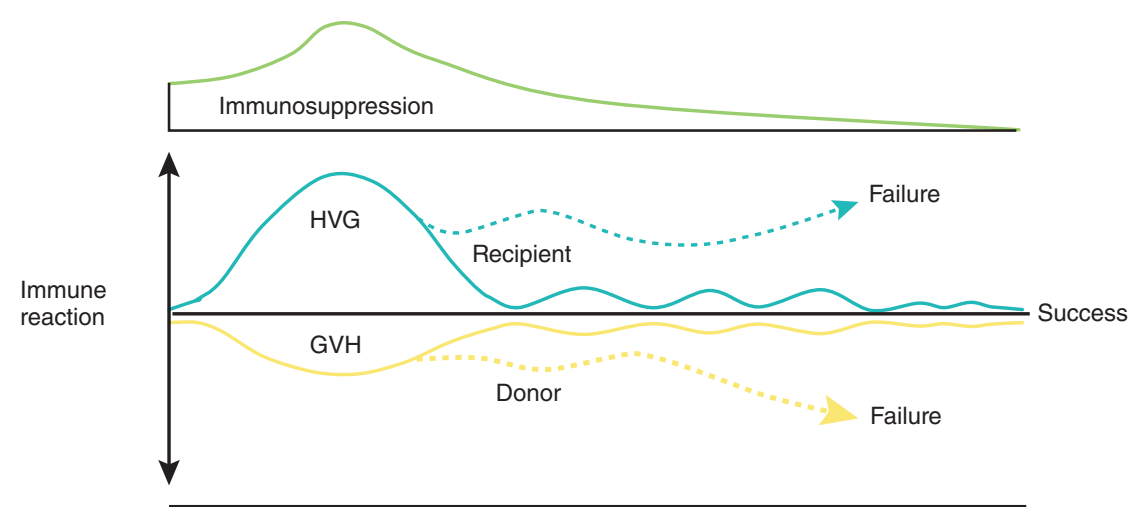

Time after organ transplantation

Figure 5 The kinetics of immunosuppression-aided exhaustion and deletion of host-versus-graft (HvG) and graft-versus-host $(\mathrm{GvH})$ responses occurring contemporaneously in organ recipients after migration of the graft's passenger leukocytes. Although host-versus-graft responses (expressed as rejection; upright curve) are dominant in most organ recipients, serious or lethal graft-versus-host responses (inverted curve), expressed as graft-versus-host disease, are not rare in recipients of lymphoid-rich organs such as liver and intestine. Therapeutic failure after organ or bone marrow transplantation implies an inability to control one or both of the responses. 